\title{
Vortex spectrum in superfluid turbulence: interpretation of a re- cent experiment
}

\author{
P.-E. Roche ${ }^{1}$ and C.F. BAREnGHI ${ }^{2}$
}

Institut NÉEL, CNRS/UJF, BP166, F-38042 Grenoble Cedex 9, France

School of Mathematics, University of Newcastle, Newcastle upon Tyne NE1 7RU, UK

PACS 67.40.Vs - Vortices and turbulence

PACS 47.37.+q - Hydrodynamic aspects of superfluidity: quantum fluids

PACS 67.57.De - Superflow and hydrodynamics in quantum fluids and solids: liquid and solid helium

\begin{abstract}
We discuss a recent experiment in which the spectrum of the vortex line density fluctuations has been measured in superfluid turbulence. The observed frequency dependence of the spectrum, $f^{-5 / 3}$, disagrees with classical vorticity spectra if, following the literature, the vortex line density is interpreted as a measure of the vorticity or enstrophy. We argue that the disagrement is solved if the vortex line density field is decomposed into a polarised field (which carries most of the energy) and an isotropic field (which is responsible for the spectrum).
\end{abstract}

Motivation and aim. - Recent experiments have explored similarities and differences between turbulence in classical, ordinary fluids and turbulence in He II (superfluid turbulence). Superfluid turbulence consists of a tangle of quantised vortex filaments; it is usually characterised (in both experiments and numerical simulations) by the vortex line density $L$ (defined as the vortex length per unit volume). Superfluid turbulence can be generated in many ways: heat currents [1-3], vibrating wires [4], - oscillating grids [5] or spheres [6], towed grids [7], bellows $[8,9]$, rotating propellers $[10,11]$ and ultrasound [12]. Superfluid turbulence is also tackled in the slightly different context of superfluid ${ }^{3} \mathrm{He}-\mathrm{B}[13,14]$.

The current understanding of superfluid turbulence at the relatively high temperature is the following. According to experimental [10,15], theoretical [16] and numerical [17] results, at sufficiently large scales in the inertial range, the normal fluid and the superfluid components of He II are strongly coupled, the superfluid and normal fluid velocities are matched, $v_{s} \sim v_{n}$, and their energy spectra obey the classical Kolmogorov law $k^{-5 / 3}$ (where the wavenumber $k$ and the frequency $f$ are related by $k=f / \bar{V}$ where $\bar{V}$ is the mean flow).

In a recent experiment, Roche et al. [11] measured the spectrum of the fluctuations of the vortex line density $L$ in turbulent superfluid helium ${ }^{4} \mathrm{He}$ at $T=1.6 \mathrm{~K}$ and found a clear $f^{-5 / 3}$ dependence. Our aim is to reconcile this observation with the current understanding of superfluid turbulence and the interpretation (which is quite common in the literature) of the vortex line density $L$ as a measure of the superfluid vorticity, $\omega_{s}=\kappa L$, where $\kappa \approx 10^{-7} \mathrm{~m}^{2} / \mathrm{s}$ is the quantum of circulation. Under this interpretation, the $f^{-5 / 3}$ spectrum observed by Roche et al. [11] seems to contradict the scaling of vorticity observed in classical turbulence, which is a flat or slowly decreasing frequency spectrum (see for example $[18,19]$ and references within).

To be more precise, what was actually measured in Ref. [11] is the vortex line density corrected by a sine squared prefactor to account for the orientation of each vortex line (as explained in Ref. [3] for example): the component of a vortex line parallel to the direction of sound propagation does not contribute to the second sound attenuation at first order. The natural interpretation of the measured signal is therefore the magnitude of the vorticity corrected by a prefactor calculated with the orientation of the vorticity vector. Using the DNS dataset 11 of Gotoh et al. [20], we have checked that the correction introduced by this prefactor has only a small contribution to the slope of the spectrum of the magnitude of the vorticity in classical turbulence. This still leaves us with a major discrepancy between the classical vorticity spectrum and the steeper $f^{-5 / 3}$ decrease which was observed.

In bringing together the current understanding of superfluid turbulence with the observed spectrum of $L$, we also need to make sure that the interpretation of all measurements performed in Ref. [11] using a pressure sensor and a second sound detector are consistent with each other. In fact, at first sight there seems to be an inconsistency between the mean vortex line density and the energy of the flow estimated from the measured velocity

For a mean velocity $\bar{V} \approx 1 \mathrm{~m} / \mathrm{s}$ at $T=1.6 \mathrm{~K}$, Roche et al. [11] report a mean vortex line density $\bar{L}$ corresponding to an average intervortex spacing $\delta$

\footnotetext{
${ }^{1}$ Data were downloaded from International Computational Fluid Dynamics database, hosted by the Cineca supercomputing center, Bologna, Italy. (http://cfd.cineca.it/)
} 


$$
\delta=1 / \sqrt{\bar{L}} \approx 4 \times 10^{-6} \mathrm{~m} .
$$

from which we estimate backwards:

$$
\bar{L}=1 / \delta^{2} \approx 6 \times 10^{10} \mathrm{~m}^{-2} .
$$

The kinetic energy per unit volume of the same flow is

$$
K=\frac{1}{2} \rho_{n} v_{n}^{2}+\frac{1}{2} \rho_{s} v_{s}^{2} .
$$

If the normal fluid and the superfluid are indeed coupled, $v_{n} \approx v_{s}$, and if we assume that this velocity is approximately $V_{r m s}=0.3 \mathrm{~m} \mathrm{~s}^{-1}$ (corresponding to the mean flow $\bar{V}=1 \mathrm{~m} \mathrm{~s}^{-1}$ and a turbulence intensity of $30 \%$ ), we have

$$
K \approx \frac{1}{2}\left(\rho_{n}+\rho_{s}\right) V_{r m s}^{2}=\frac{\rho}{2} V_{r m s}^{2}=6.5 \mathrm{~J} \mathrm{~m}^{-3} .
$$

where $\rho=\rho_{s}+\rho_{n}=145 \mathrm{~kg} \mathrm{~m}^{-3}$. Since $\rho_{n}<0.2 \rho_{s}$, this energy $K$ is approximately equal to the superfluid kinetic energy. Let us assume that the kinetic energy per unit volume, $K$, is approximately equal to the kinetic energy per unit length, $\mathcal{E}$, times the length per unit volume $L$ :

$$
K \approx \mathcal{E} L .
$$

The kinetic energy per unit length is obtained in cylindrical coordinates $(r, \phi, z)$ by integrating the square of the velocity field $\kappa /(2 \pi r)$ around a straight vortex line (set along the $z$ direction) from the radial distance $r=a \approx 10^{-10} \mathrm{~m}$ (the vortex core radius) to some upper cutoff $b$ :

$$
\mathcal{E}=\frac{\rho_{s}}{2} \int_{0}^{2 \pi} d \phi \int_{a}^{b} r\left(\frac{\kappa}{2 \pi r}\right)^{2} d r=\frac{\rho_{s} \kappa^{2}}{4 \pi} \ln (b / a) .
$$

If we take $b=\delta$, using $\rho_{s}=122 \mathrm{~kg} \mathrm{~m}^{-3}$ at $T=1.6 \mathrm{~K}$, we have

$$
\mathcal{E} \approx 1.0 \times 10^{-12} \mathrm{~J} \mathrm{~m}^{-1},
$$

thus

$$
L \approx K / \mathcal{E}=6 \times 10^{12} \mathrm{~m}^{-2},
$$

which is much bigger than the value of $\bar{L}$ from second sound measurements, $L=6 \times 10^{10} \mathrm{~m}^{-2}$. This second apparent inconsistency must be solved too.

The following model which we propose to solve these inconsistencies may not be the final answer. Nevertheless, we think that the exercise of putting together a coherent scenario with the experimental information which is available at this stage is a valuable exercise which should stimulate further work and bring us closer to the correct solution of the puzzle.

Model. - The model which we propose to solve the puzzle described in the previous section has two key features: the decomposition of the vortex line distribution into a "polarised" field and a "unpolarised" (or "isotropic") field, and the assumption that the unpolarised field has some statistical properties of a passive vector field. We stress that our interpretation is preliminary.
Decomposition of the vortex line density. Since the vortex core radius is many orders of magnitude smaller than $\delta$ or any other length scale of interest in the flow, we follow Schwarz [21] and describe vortex lines as space curves $\mathbf{s}(\xi, t)$ where $\xi$ is arclength and $t$ is time. The quantity $\mathbf{s}^{\prime}=d \mathbf{s} / d \xi$ is the unit vector at the point $\mathbf{x}=\mathbf{s}$ in the tangent direction along the vortex line.

Consider a small cubic box $\Delta(\mathbf{x})$ of size $\Delta>\delta$ and volume $\Delta^{3}$ centred around the point $\mathbf{x}$. We define the coarse-grained superfluid vorticity field as

$$
\boldsymbol{\omega}_{s}(\mathbf{x})=\frac{\kappa}{\Delta^{3}} \int_{\Delta(\mathbf{x})} \mathbf{s}^{\prime} d \xi
$$

This definition corresponds to the same coarse-graining procedure which was used in Ref [22] in a numerical calculation of a vortex tangle driven by an $\mathrm{ABC}$ normal flow to show that the (coarse-grained) superfluid vorticity matches the vorticity of the normal flow.

Note that $\boldsymbol{\omega}_{s}$ is nonzero only if the vortex lines are spatially organised. If the vortex lines point randomly in all directions, then each Cartesian component of $\boldsymbol{\omega}_{s}$ is zero, because in each direction oriented vortex strands cancel each other out when summed algebraically.

It is easy to check that the magnitude of $\boldsymbol{\omega}_{s}$ is less than $\kappa$ times the local vortex line density $L$ :

$$
\begin{array}{r}
\left|\boldsymbol{\omega}_{s}(\mathbf{x})\right|=\left|\frac{\kappa}{\Delta^{3}} \int_{\Delta(\mathbf{x})} \mathbf{s}^{\prime} d \xi\right| \\
<\frac{\kappa}{\Delta^{3}} \int_{\Delta(\mathbf{x})}\left|\mathbf{s}^{\prime}\right| d \xi=\frac{\kappa}{\Delta^{3}} \int_{\Delta(\mathbf{x})} d \xi=\kappa L(\mathbf{x}),
\end{array}
$$

because $\left|\mathbf{s}^{\prime}\right|=1$.

From the coarse-grained superfluid vorticity we can define the (local) polarised vortex line density $L_{\|}$:

$$
\kappa L_{\|}(\mathbf{x})=\left|\boldsymbol{\omega}_{s}(\mathbf{x})\right|,
$$

Since

$$
L_{\|}(\mathbf{x})<L(\mathbf{x}) .
$$

the missing part is a field which we call $L_{\times}(\mathbf{x})$ and we have the (local) decomposition

$$
L(\mathbf{x})=L_{\times}(\mathbf{x})+L_{\|}(\mathbf{x}) .
$$

The smoothed field $L_{\|}$filters the vortex tangle in $k$ space, getting rid of short-wavelength Kelvin waves on the same vortex line. It also accounts for cancellation effects arising from vortex lines oriented in opposing directions. By construction, $L_{\|}$is sufficiently organised that it defines the coarse-grained superfluid vorticity field $\boldsymbol{\omega}_{s}$. Thus $L_{\|}$ reflects the superfluid velocity field in the inertial range at scales larger than $\Delta$. At scales smaller than $\Delta$ the superfluid vorticity field $L_{\|}$is clearly not defined.

Viceversa, $L_{\times}=L-L_{\|}$contributes to the vortex line density but not to the superfluid vorticity and reflects the randomly oriented vortex lines. Note that we have not made any assumption about the relative amount of 
wiggliness of $L_{\|}$and $L_{\times}$. Notice that $L_{\times}$does not necessarily consist only of small loops (left over by vortex reconnections for example) or high energy Kelvin waves $\left(k \gg \Delta^{-1}\right)$. Long filaments, provided they are randomly oriented with respect to their neighbours (so that they do not add up vortex length in the same direction), can be part of $L_{\times}$.

Passive vectors. The second feature of our model is the assumption that the unpolarised field $L_{\times}$has some statistical properties of a classical passive vector field. It is well known [24] that passive vectors have a power spectrum which obeys the $f^{-5 / 3}$ law, and this is our explanation of the observed power spectrum of the vortex line density.

At first it may seem contradictory to expect $L_{\times}$to correspond to a active field while assuming a passive nature for $L_{\|}$. We now argue that this active/passive distinction may result from a fundamental property of superfluid vortices which makes them different from classical vortices.

In a classical fluid [25] the local time derivative of the vorticity is the combination of advection, $(\mathbf{v} \cdot \nabla) \boldsymbol{\omega}$, and stretching, $(\boldsymbol{\omega} \cdot \nabla) \mathbf{v}$. An example of the latter is the stretching of a classical vortex along its main axis which elongates it while squeezing it transversally, resulting in an increase of vorticity. In the superfluid vortex stretching does not occur because the radius of the vortex core is fixed, determined by quantum mechanical constraints on the rotation. Superfluid vortices can become longer (for example, if energy is fed from the normal fluid, or, at $T=0$, if the geometry changes keeping the total kinetic energy constant), but their core is rigid, thus they remain slender with respect to any typical turbulence scale. Superfluid vortex dynamics thus differs from the dynamics of classical vortices (for example see $[25,26]$ ). Nevertheless, when superfluid vortices are assembled in polarised bundles, the classical vorticity enhancement that is described above can still be reproduced by stretching the whole bundle, as if vortices were material lines. For example, axisymmetric and non-axisymmetric oscillations of superfluid vortex bundles in the form of waves are known in the literature [27]. It is therefore reasonable to expect that the field $\boldsymbol{\omega}_{s}$, which result from polarised vortices, will mimic a classical active vorticity field at large enough scales.

We now turn to the stretching of an unpolarised tangle by a large scale velocity field. If, as modelled above, superfluid vortices behave as material lines, it is also reasonable to expect that the tangle will remain unpolarised, and, due to the fluid incompressibility, the total length of lines will remain unchanged : in other words, $L_{\|}$will be simply transported by the coarse-grained velocity.

Viceversa, it is reasonable to assume that the high density and large density fluctuations of $L_{\times}$have little impact on the dynamics of the polarised field $L_{\|}$or $\boldsymbol{\omega}_{s}$, that is to say that $L \times$ does not advect $L_{\|}$at scale large than $\Delta$ (where $L_{\|}$is defined). This must be the case, because the velocity field induced by the unpolarised field must be very short-ranged, probably $1 / r^{2}$, caused by multipolar sources with no contribution at first order $1 / r$.

\section{Consistency with measurements. -}

The spectrum of the polarised field. Our model is consistent only if we can show that the polarised vortex line density $L_{\|}$gives a negligible contribution to the spectrum below $1 \mathrm{kHz}$, which is the observed second sound frequency range used by Roche et al.

Following what said in the introduction, we approximate the second sound spectrum $P_{\|}$arising from $L_{\|}$as a white noise signal up to a viscous cutoff corresponding to few times the frequency of the Kolmogorov length scale $\eta$ :

$$
P_{\|}=\frac{\overline{L_{\|}^{2}}-{\overline{L_{\|}}}^{2}}{\bar{V} /(4 \eta)}
$$

The denominator is the full frequency span of an ideal second sound probe. Eq14 represents the (constant) power spectral density at all frequencies below the cut-off frequency. An ideal second sound probe is fixed in space. The smallest time scales which are visible to the probe are produced by the smallest flow structures (of typical size $4 \eta$ ) which are advected past the probe at the local fluid velocity (approximated by the mean flow velocity). The inverse of this time scale gives the highest frequency of the signal seen by the ideal probe, which is indeed the frequency span.

Let us find an upper bound for $P_{\|}$. In classical turbulence, both experimental [23] and numerical studies suggest that the vorticity $\boldsymbol{\omega}$ roughly satisfies

$$
\overline{\omega^{2}} \sim 2 \overline{|\omega|}^{2},
$$

If we assume that this classical relation applies to the the normal fluid in the experiment [11], and if we make the further assumptions that normal fluid and superfluid are locked, $\omega_{s} \approx \omega_{n}$, and that $\kappa L_{\|}=\omega_{s}$, we have

$$
\overline{L_{\|}^{2}} \simeq 2{\overline{L_{\|}}}^{2}
$$

Thus

$$
P_{\|} \sim \frac{2 \eta}{\bar{V}} \overline{L_{\|}^{2}}=\frac{2 \eta}{\bar{V}}\left(\frac{\mu \kappa^{2} \overline{L_{\|}^{2}}}{\mu \kappa^{2}}\right),
$$

where $\nu=\mu / \rho$ is the kinematic viscosity and $\mu$ the viscosity of helium.

A bound for $P_{\|}$can be found by noticing that the total rate of dissipation of kinetic energy per unit volume in turbulent He II, $\rho \epsilon$, should be larger than the dissipation $\mu \omega_{n}^{2}$ which arises from the regular viscous dissipation in the normal fluid alone:

$$
\mu \omega_{n}^{2}<\rho \epsilon,
$$

where, since $\omega_{s} \approx \omega_{n}$,

$$
\mu \omega_{n}^{2} \approx \mu \omega_{s}^{2}=\mu \kappa^{2} \overline{L_{\|}^{2}},
$$


Using the kinematic viscosity $\nu=\mu / \rho$, we find

$$
P_{\|}<\frac{2 \eta}{\bar{V}}\left(\frac{\epsilon}{\nu \kappa^{2}}\right)
$$

In the next section, we present different ways of evaluating the Kolmogorov length $\eta$ at $T=1.6 \mathrm{~K}$ and show that they all give the same order of magnitude for $\eta$. For clarity, we use the following classical expression (with the kinematic viscosity defined above):

$$
\eta \approx\left(\nu^{3} / \epsilon\right)^{1 / 4} .
$$

We have

$$
P_{\|}<\frac{2}{\kappa^{2} \bar{V}}\left(\frac{\epsilon^{3}}{\nu}\right)^{1 / 4},
$$

To evaluate this expression, we estimate the rate of kinetic energy dissipation $\epsilon$ at the integral scale, $\ell_{0} \approx$ $10^{-2} \mathrm{~m}$, for which $V_{0} \approx V_{r m s}=0.3 \mathrm{~m} / \mathrm{s}$, and obtain

$$
\epsilon \approx \frac{V_{0}^{3}}{\ell_{0}} \approx \frac{V_{r m s}^{3}}{\ell_{0}} \approx 2.7 \mathrm{~m}^{2} \mathrm{~s}^{-3},
$$

At $T=1.6 \mathrm{~K}, \nu \approx 8.9 \times 10^{-9} \mathrm{~m}^{2} \mathrm{~s}^{-1}$ and we obtain $P_{\|}<4.3 \times 10^{16} \mathrm{~m}^{-4} \mathrm{~s}$, which is much less then the observed spectral density $P=2 \times 10^{19} \mathrm{~m}^{-4} \mathrm{~s}$ and is just above the instrumental noise level $0.5 \times 10^{16} \mathrm{~m}^{-4} \mathrm{~s}$ (see Fig 4 of Ref. [11]). We conclude that the contribution of $L_{\|}$to the observed spectrum is negligible.

The Kolmogorov length. We estimate the Kolmogorov length from the expression $\eta=\left(\nu^{\star 3} / \epsilon\right)^{1 / 4}$. In principle we can define three possible kinematic viscosities $\nu^{\star}$ in our problem (all numerical values refer to $T=1.6 \mathrm{~K}$ ). The first is based on the total density $\rho=\rho_{n}+\rho_{s}=145 \mathrm{~kg} \mathrm{~m}^{-3}$ and is $\nu=\mu / \rho=8.9 \times 10^{-9} \mathrm{~m}^{2} \mathrm{~s}^{-1}$. The second is based on the normal fluid density $\rho_{n}=23.6 \mathrm{~kg} \mathrm{~m}^{-3}$ and is $\nu_{n}=\mu / \rho_{n}=55 \times 10^{-9} \mathrm{~m}^{2} \mathrm{~s}^{-1}$. The third is the efficient kinematic viscosity $\nu^{\prime} \approx 20 \times 10^{-9} \mathrm{~m}^{2} \mathrm{~s}^{-1}$ determined from towed-grid experiments in turbulent He II, see fig. 8 of Ref . [15] Using these three values, we obtain respectively $\eta \approx 0.7 \mu \mathrm{m}, \eta \approx 3 \mu \mathrm{m}$ and $\eta \approx 1 \mu \mathrm{m}$. In all cases $\eta$ is of the order of magnitude of the intervortex spacing, $\delta \approx 4 \mu \mathrm{m}$ or at the most six times smaller. This is consistent with the argument of Vinen and Niemela [16] that $\delta$ and $\eta$ are likely to be of the same order of magnitude: the superfluid and the normal fluid are coupled throughout the inertial range.

The energy. A key feature of our model is that the unpolarised field $L_{\times}$gives a negligible contribution to the total energy of the flow compared to the polarised field $L_{\|}$. The estimate made in the introduction that the kinetic energy per unit volume is $K \approx 6.5 \mathrm{Jm}^{-3}$ clearly refers to the polarised field $L_{\|}$, because we obtained it using the condition $v_{n} \approx v_{s}$ : in this notation we rewrite

$$
\begin{array}{r}
K_{\|}=\frac{1}{2} \rho_{n} v_{n}^{2}+\frac{1}{2} \rho_{s} v_{s}^{2} \approx \frac{1}{2}\left(\rho_{n}+\rho_{s}\right) V_{r m s}^{2} \\
=\frac{\rho}{2} V_{r m s}^{2}=6.5 \mathrm{~J} \mathrm{~m}^{-3}
\end{array}
$$

Let us estimate the energy contained in the unpolarised field $L_{\times}$. If we picture $L_{\times}$as a random network of straight vortex lines, the energy per unit volume can be obtained following the integral procedure that leads to Eq 6 with $b=\delta$ : we multiply the length per unit volume times the integral of the square of the velocity field only up to a radial distance which is of the order of the intervortex spacing, because at this distance the total velocity fields of randomly oriented vortices cancel each other out. Using the numerical value provided in $\mathrm{Eq} 6$ and $\mathrm{Eq} 2$ for $L_{\times} \approx \bar{L}$, we get

$$
K_{\times} \approx \frac{\rho_{s} \kappa^{2}}{4 \pi} L_{\times} \ln (\delta / a) \approx 0.06 \mathrm{Jm}^{-3}<<K_{\|} .
$$

The above integration procedure would not give the kinetic energy per unit volume arising from the polarised field $L_{\|}$, because contributions of different vortex lines add up rather than cancel each other. This why we expect $K_{\|}>>K_{\times}$to hold when a sufficient level of vortex polarisation is reached.

The argument is made more clear if we consider a cylindrical container of radius $h$ and height $h$ containing $N$ straight vortex lines aligned along the axis. The number of vortex lines per unit area is $L=N /\left(\pi h^{2}\right)$ and the intervortex distance is $\delta=\sqrt{\pi h^{2} / N}$. Suppose that the vortices are oriented in the same direction (complete polarisation), forming a vortex bundle, as in a recent numerical calculation [28]; then the velocity fields of the vortices add up and create a total solid-body rotation velocity $v=\Omega r$ where $\Omega$ is obtained from

$$
N \kappa=\oint_{C} \mathbf{v} \cdot \mathbf{d} \ell=\int_{S} \nabla \times \mathbf{v} \cdot \mathbf{d} \mathbf{S}=\int_{S} \boldsymbol{\omega} \cdot \mathbf{d} \mathbf{S}=2 \Omega \pi h^{2},
$$

which yields

$$
\Omega=N \kappa /\left(2 \pi h^{2}\right) .
$$

The energy per unit volume is

$$
E_{\|}=\frac{\rho_{s} \kappa^{2} h^{2} L^{2}}{16},
$$

Now assume the opposite limit, that the vortices are randomly oriented in the positive or negative direction along the axis of the cylinder. The energy per unit volume is

$$
\begin{array}{r}
E_{\times}=\frac{1}{\pi h^{3}} \frac{\rho_{s}}{2} N \int_{0}^{h} d z \int_{0}^{2 \pi} d \phi \int_{a}^{\delta} d r r\left(\frac{\kappa}{2 \pi r}\right)^{2} \\
=\frac{\rho_{s} \kappa^{2} L}{8 \pi} \ln \left(1 /\left(L a^{2}\right)\right),
\end{array}
$$

(We write it in terms of $L$ rather than $N$ because we want to take the limit of increasing the density rather than the number of vortices). Therefore

$$
\frac{E_{\times}}{E_{\|}}=\frac{2}{\pi} \frac{1}{L h^{2}} \ln \left(1 /\left(L a^{2}\right)\right)=\frac{4}{\pi} \frac{\delta^{2}}{h^{2}} \ln (\delta / a),
$$

Clearly, the larger is the vortex line density $L$, the smaller is $E_{\times}$with respect to $E_{\|}$(provided $\delta>a$ of course). 
Conclusion. - Our model of superfluid turbulence consists in dividing the tangle in a polarised field $L_{\|}$and a unpolarised field $L_{\times}$such that the total vortex line density is $L=L_{\|}+L_{\times}$. The polarised field makes up the (coarse-grained) superfluid vorticity field, such that the two fluids are coupled $\left(v_{n} \sim v_{s}\right)$ in the inertial range according to current understanding of superfluid turbulence. The unpolarised field has some of the statistical properties of a passive vector field. What is observed with the second sound probe is mainly $L_{\times}$, not $L_{\|}$, which, as we have shown, brings a negligible contribution to the measured spectrum:

$$
P_{\times}>>P_{\|} .
$$

However the polarised field $L_{\|}$has more energy than $L_{\times}$:

$$
K_{\|}>>K_{\times}
$$

That is why if we try to infer the vortex line density from $\mathrm{Eq} 8$ we get an unrealistic high value.

The model suggests the following picture of the turbulent tangle in the large scales (upper inertial range): large fluctuations of the density of vortex line on top of a small modulation of polarisation which -nevertheless- controls the velocity field, energy cascade and passive advection of most of the vortex line density.

We stress that the model which we propose is only an attempt to combine the information which is available from the experiment of Roche et al. [11] in a consistent scenario. If the model can be confirmed, the $f^{-5 / 3}$ power law dependence of the fluctuations of the vortex line density reported in [11] should be considered as the inertial-range signature of the quantum nature of superfluid turbulence.

Finally we remark that a decomposition of the vortex line density field in polarised and isotropic parts similar in spirit to what we have done, has been attempted by Lipniacki [29]. Lipniacki's theory results in an Euler equation (motified by the presence of friction) which is similar to Hall-Vinen equation for the macroscopic superfluid velocity [30], which is coupled to a modified Vinen equation for the (more microscopic and isotropic) vortex line density $L$.

C.F.B. is supported by EPSRC grants GR/T08876/01 and EP/D040892/1 and P.-E. R. by the ANR grant TSF.

\section{REFERENCES}

[1] Zhang T. and Van Sciver S., Nature Physics, 1 (36) 2005

[2] Zhang T. and Van Sciver S., J. Low Temp. Physics, 138 (865) 2005

[3] Barenghi C.F., Gordeev A.V. and Skrbek L., Phys. Rev. E, 74 (026309) 2006

[4] Blazkova M., Schmoranzer D and Skrbek L., Phys. Rev. E, 75 (025302) 2007
[5] Charambolous D., Skrbek L, Hendry P.C.,McClintock P.V.E. and Vinen W.F., Phys. Rev. E, 74 (036307) 2006

[6] Jager J., Schuderer B. and Schoepe W., Phys. Rev. Lett., 74 (566) 1995

[7] Stalp S.R., Skrbek L. and Donnelly R.J., Phys. Rev. Lett., 82 (4831) 1999

[8] Fuzier S., Baudouy B. and Van Sciver S. W., Cryogenics, 41 (2001) 453.

[9] Smith M.R. , Hilton D.K. and Van Sciver S. W., Phys. Fluids, 11 (1999) 751.

[10] Maurer J. and Tabeling P., Europhysics Lett., 43 (1998) 29.

[11] Roche P.-E., Diribarne P., Didelot T., Français O., Rousseau L. and Willaime H., EPL, 77 (2007) 66002.

[12] Milliken F.P., Schwarz K.W. and Smith C.W., Phys. Rev. Lett., 48 (1982) 1204.

[13] Finne A.P., Araki T., Blaauwgeers R., Eltsov V.B., Kopnin N.B., Krusius M., Skrbek L., Tsubota M. and Volovik G.E., Nature, 424 (1022) 2003

[14] Bradley D.I., Clubb D.O., Fisher S.N., Guenault A.M., Haley R.P., Matthews C.J., Pickett G.R., Tsepelin V. and Zaki K., Phys. Rev. Lett., 93 (035302) 2005

[15] Stalp S. Niemela J.J., Vinen W.F., and Donnelly R.J., Phys. Fluids, 14 (2002) 1377.

[16] Vinen W.F. and Niemela J.J., J. Low Temp. Phys., 128 (2002) 128.

[17] Kivotides D., Vassilicos J.C., Samuels D.C. and BARENGHI C.F., Europhys. Lett., 57 (2002) 845.

[18] Ishihara T., Kaneda Y., Yokokawa M., Itakura K. and Uno A., J. Phys. Soc. Japan, 72 (2003) 983.

[19] Zhou T., Antonia R.A. and Chua L.P., J. of Turbulence, 6 (2005) 28.

[20] Gotoh T., Fukayama D. and Nakano T., Phys. Fluids, 14 (2002) 1065.

[21] Schwarz K.W., Phys. Rev. B, 38 (1988) 2398.

[22] Barenghi C.F., Samuels D.C. and Bauer G.H., Phys. Fluids, 9 (1997) 2631.

[23] Antonia R.A., Zhou T. and Zhou Y., J. Fluid Mech., 374 (1998) 29.

[24] Ohkitani K., Phys. Rev. E, 65 (2002) 046304.

[25] Chorin, A., Vorticity and Turbulence, (1997) Springer 2nd ed..

[26] Zhou, H., Phys. Fluids, 9 (1997) 970.

[27] Henderson K.L. And Barenghi C.F., Europhys Lett., 67 (2004) 56.

[28] Kivotides D., Phys. Rev. Lett., 96 (2007) 175301.

[29] Lipniacki T., European J. Mech. B-Fluids, 25 (2006) 435

[30] Henderson K.L., Barenghi C.F. and Jones C.A., J. Fluid Mech., 283 (1995) 329. 